\title{
Crystal structure of $\left(1 S^{*}, 2 R^{*}, 9 R^{*}, 10 S^{*}\right)-2,10$-diacetoxi-13-oxa- bicyclo[7.3.1]tridec-5-ene, $\mathrm{C}_{16} \mathrm{H}_{24} \mathrm{O}_{5}$
}

\section{Brito Bobadilla}

Universidad de Antofagasta, Facultad de Ciencias Básicas, Departamento de Química, casilla 170, Antofagasta, Chile

\section{O. Wittke Gunter}

Universidad de Chile, Facultad de Ciencias Físicas y Matemáticas, Departamento de Física, casilla 487-3, Santiago, Chile

\section{J. D. Martin and I. Brouard}

Instituto de Investigaciones Químicas, CSIC, Americo Vespucio, S/N, Isla de La cartuja 41092 Sevilla, Spain

Received July 3, 1998, CSD-No. 409349

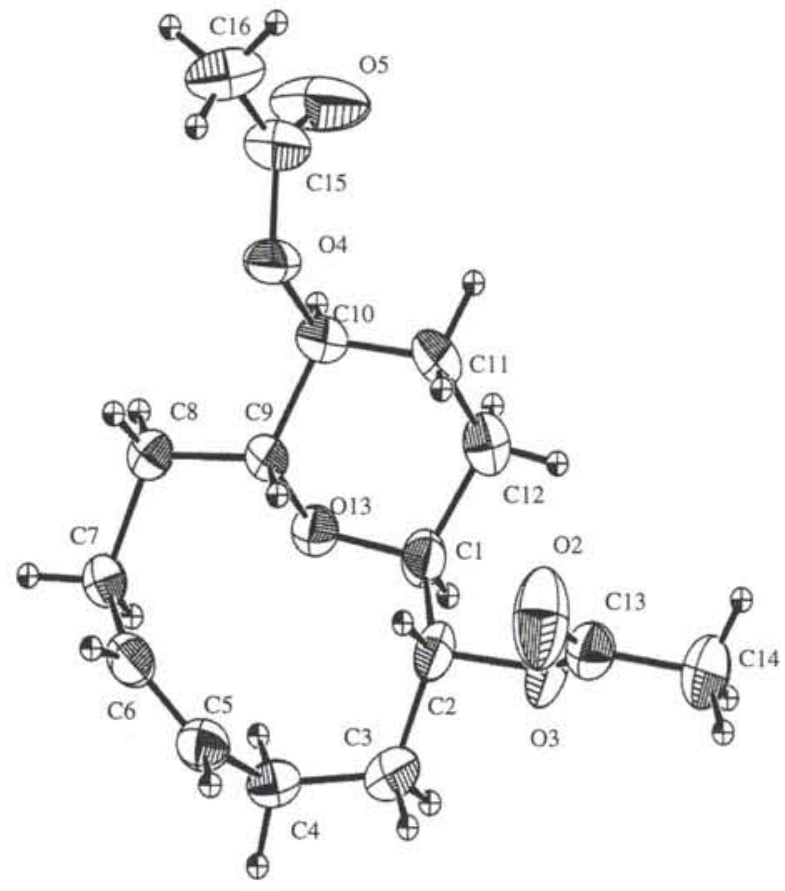

Table 1. Data collection and handling.

$\begin{array}{ll}\text { Crystal: } & \text { colorless prism, size } 0.15 \times 0.16 \times 0.12 \mathrm{~mm} \\ \text { Wavelength: } & \text { Mo } K_{\alpha} \text { radiation }(0.71073 \AA) \\ \mu: & 0.89 \mathrm{~cm}^{-1} \\ \text { Diffractometer, scan mode: } & \text { Siemens R3m/V, } \omega \\ 2 \theta_{\text {max: }} & 62^{\circ} \\ N(h k l)_{\text {measured }}, N(h k l)_{\text {unique: }} & 5496,5220 \\ \text { Criterion for } F_{\text {obs }}, N(h k l)_{\text {gt: }}: & F_{\text {obs }}>3 \sigma\left(F_{\text {obs }}\right), 1365 \\ N(\text { param })_{\text {refined: }} & 190 \\ \text { Programs: } & \text { SHELXS-86 [4], SHELXL-93 [5] }\end{array}$

\section{Abstract}

$\mathrm{C}_{16} \mathrm{H}_{24} \mathrm{O}_{5}$, monoclinic, $P 12{ }_{1} / c 1$ (No. 14), $a=14.941$ (2) $\AA$, $b=11.213(2) \AA, c=9.888(2) \AA, \beta=101.4(1)^{\circ}, V=1623.9 \AA^{3}$, $Z=4, R_{\mathrm{gt}}(F)=0.051, R_{\mathrm{w}}\left(F^{2}\right)=0.113, T=293 \mathrm{~K}$.

\section{Source of material}

The compound was prepared using literature procedures [1-3].

\section{Discussion}

The molecular structure of this compound possesses a ten-membered ring trans-fused with a six-menbered ring, and two acetoxygroup. In the cyclodecane part of the molecule, there is only $\mathrm{C}=\mathrm{C}$ double bond, namely $\mathrm{C} 5=\mathrm{C} 6$; all the other $\mathrm{C}-\mathrm{C}$ bond lenghts have values in the simple bond range, with $\mathrm{C} 4-\mathrm{C} 5$ and $\mathrm{C} 6-\mathrm{C} 7$ being $\mathrm{Csp}^{2}-\mathrm{Csp}^{3}$ bonds, while the rest are $\mathrm{Csp}^{3}-\mathrm{Csp}^{3}$ and $\mathrm{Csp}^{3}-\mathrm{O}$. The six-membered ring exhibits a slightly distorted chair conformation. All distances and angles are normal.

Table 2. Atomic coordinates and displacement parameters (in $\AA^{2}$ ).

\begin{tabular}{|c|c|c|c|c|c|}
\hline Atom & Site & $x$ & $y$ & $z$ & $U_{\text {iso }}$ \\
\hline $\mathrm{H}(1)$ & $4 e$ & $0.2117(2)$ & $0.5265(2)$ & $-0.2805(3)$ & 0.062 \\
\hline $\mathbf{H}(2)$ & $4 e$ & $0.1820(2)$ & $0.5567(2)$ & $-0.0084(2)$ & 0.060 \\
\hline $\mathrm{H}(3 \mathrm{~A})$ & $4 e$ & $0.0319(2)$ & $0.4990(2)$ & $-0.1006(3)$ & 0.069 \\
\hline $\mathrm{H}(3 \mathrm{~B})$ & $4 e$ & $0.0546(2)$ & $0.4879(2)$ & $-0.2477(3)$ & 0.069 \\
\hline$H(4 A)$ & $4 e$ & $0.0644(2)$ & $0.6944(2)$ & $-0.2644(2)$ & 0.062 \\
\hline $\mathrm{H}(4 \mathrm{~B})$ & $4 e$ & $-0.0316(2)$ & $0.6579(2)$ & $-0.2373(2)$ & 0.062 \\
\hline $\mathrm{H}(5)$ & $4 e$ & $0.0176(1)$ & $0.7215(2)$ & $-0.0037(2)$ & 0.061 \\
\hline$H(6)$ & $4 e$ & $0.0913(2)$ & $0.8874(2)$ & $0.0200(2)$ & 0.062 \\
\hline $\mathrm{H}(7 \mathrm{~A})$ & $4 e$ & $0.1300(1)$ & $0.9842(2)$ & $-0.1677(2)$ & 0.062 \\
\hline $\mathrm{H}(7 \mathrm{~B})$ & $4 e$ & $0.1233(1)$ & $0.8640(2)$ & $-0.2500(2)$ & 0.062 \\
\hline $\mathrm{H}(8 \mathrm{~A})$ & $4 e$ & $0.2769(1)$ & $0.9106(2)$ & $-0.1888(3)$ & 0.062 \\
\hline $\mathrm{H}(8 \mathrm{~B})$ & $4 e$ & $0.2698(1)$ & $0.9307(2)$ & $-0.0343(3)$ & 0.062 \\
\hline $\mathrm{H}(9)$ & $4 e$ & $0.2478(1)$ & $0.7281(2)$ & $-0.0044(2)$ & 0.053 \\
\hline $\mathrm{H}(10)$ & $4 e$ & $0.3979(1)$ & $0.7448(2)$ & $-0.1424(3)$ & 0.064 \\
\hline $\mathrm{H}(11 \mathrm{~A})$ & $4 e$ & $0.3644(2)$ & $0.5758(2)$ & $0.0567(3)$ & 0.084 \\
\hline $\mathrm{H}(11 \mathrm{~B})$ & $4 e$ & $0.4507(2)$ & $0.5738(2)$ & $-0.0120(3)$ & 0.084 \\
\hline $\mathrm{H}(12 \mathrm{~A})$ & $4 e$ & $0.3356(2)$ & $0.4371(2)$ & $-0.1200(3)$ & 0.085 \\
\hline $\mathrm{H}(12 \mathrm{~B})$ & $4 e$ & $0.3630(2)$ & $0.5281(2)$ & $-0.2252(3)$ & 0.085 \\
\hline $\mathrm{H}(14 \mathrm{~A})$ & $4 e$ & $0.2019(2)$ & $0.1840(2)$ & $-0.0576(3)$ & 0.126 \\
\hline $\mathrm{H}(14 \mathrm{~B})$ & $4 e$ & $0.1758(2)$ & $0.1729(2)$ & $0.0878(3)$ & 0.126 \\
\hline $\mathrm{H}(14 \mathrm{C})$ & $4 e$ & $0.2781(2)$ & $0.1896(2)$ & $0.0762(3)$ & 0.126 \\
\hline $\mathrm{H}(16 \mathrm{~A})$ & $4 e$ & $0.5030(2)$ & $0.9080(3)$ & $0.2392(3)$ & 0.134 \\
\hline$H(16 B)$ & $4 e$ & $0.5700(2)$ & $0.9682(3)$ & $0.1562(3)$ & 0.134 \\
\hline $\mathrm{H}(16 \mathrm{C})$ & $4 e$ & $0.5983(2)$ & $0.8499(3)$ & $0.2379(3)$ & 0.134 \\
\hline
\end{tabular}


Table 3. Atomic coordinates and displacement parameters (in $\AA^{2}$ ).

\begin{tabular}{|c|c|c|c|c|c|c|c|c|c|c|}
\hline Atom & Site & $x$ & $y$ & $z$ & $U_{11}$ & $U_{22}$ & $U_{33}$ & $U_{12}$ & $U_{13}$ & $U_{23}$ \\
\hline$O(13)$ & $4 e$ & $0.22940(9)$ & $0.6867(1)$ & $-0.2040(2)$ & $0.0435(9)$ & $0.0451(9)$ & $0.0496(9)$ & $-0.0010(7)$ & $0.0040(7)$ & $0.0003(8)$ \\
\hline$O(2)$ & $4 e$ & $0.1810(1)$ & $0.3891(1)$ & $-0.0839(2)$ & $0.106(1)$ & $0.0330(9)$ & $0.060(1)$ & $-0.0055(9)$ & $0.007(1)$ & $-0.0023(9)$ \\
\hline$O(3)$ & $4 e$ & $0.2180(2)$ & $0.3997(2)$ & $0.1434(2)$ & $0.173(2)$ & $0.068(1)$ & $0.063(1)$ & $0.023(1)$ & $0.002(1)$ & $0.003(1)$ \\
\hline$O(4)$ & $4 e$ & $0.4199(1)$ & $0.7964(1)$ & $0.0556(2)$ & $0.0342(9)$ & $0.069(1)$ & $0.066(1)$ & $-0.0073(8)$ & $0.0012(8)$ & $-0.0035(9)$ \\
\hline$O(5)$ & $4 e$ & $0.5476(1)$ & $0.7917(3)$ & $-0.0263(3)$ & $0.056(1)$ & $0.208(3)$ & $0.125(2)$ & $-0.037(2)$ & $0.034(1)$ & $-0.060(2)$ \\
\hline$C(1)$ & $4 e$ & $0.2341(2)$ & $0.5598(2)$ & $-0.1884(3)$ & $0.057(2)$ & $0.041(1)$ & $0.055(2)$ & $0.001(1)$ & $0.010(1)$ & $-0.004(1)$ \\
\hline$C(2)$ & $4 e$ & $0.1679(2)$ & $0.5179(2)$ & $-0.0990(2)$ & $0.064(2)$ & $0.031(1)$ & $0.052(1)$ & $-0.004(1)$ & $0.005(1)$ & $-0.002(1)$ \\
\hline$C(3)$ & $4 e$ & $0.0674(2)$ & $0.5338(2)$ & $-0.1629(3)$ & $0.056(2)$ & $0.058(2)$ & $0.060(2)$ & $-0.016(1)$ & $0.014(1)$ & $-0.006(1)$ \\
\hline$C(4)$ & $4 e$ & $0.0329(2)$ & $0.6613(2)$ & $-0.1961(2)$ & $0.042(1)$ & $0.058(2)$ & $0.052(2)$ & $-0.009(1)$ & $0.001(1)$ & $-0.004(1)$ \\
\hline$C(5)$ & $4 e$ & $0.0453(1)$ & $0.7437(2)$ & $-0.0762(2)$ & $0.045(1)$ & $0.058(2)$ & $0.050(1)$ & $-0.001(1)$ & $0.013(1)$ & $-0.002(1)$ \\
\hline$C(6)$ & $4 e$ & $0.0914(2)$ & $0.8453(2)$ & $-0.0610(2)$ & $0.044(1)$ & $0.054(2)$ & $0.054(2)$ & $0.009(1)$ & $0.005(1)$ & $-0.005(1)$ \\
\hline$C(7)$ & $4 e$ & $0.1436(1)$ & $0.8996(2)$ & $-0.1597(2)$ & $0.044(1)$ & $0.042(1)$ & $0.065(2)$ & $0.005(1)$ & $0.003(1)$ & $0.010(1)$ \\
\hline $\mathrm{C}(8)$ & $4 e$ & $0.2475(1)$ & $0.8828(2)$ & $-0.1156(3)$ & $0.044(1)$ & $0.042(1)$ & $0.069(2)$ & $-0.003(1)$ & $0.008(1)$ & $0.008(1)$ \\
\hline $\mathrm{C}(9)$ & $4 e$ & $0.2724(1)$ & $0.7539(2)$ & $-0.0844(2)$ & $0.035(1)$ & $0.040(1)$ & $0.056(1)$ & $0.002(1)$ & $0.002(1)$ & $0.002(1)$ \\
\hline$C(10)$ & $4 e$ & $0.3737(1)$ & $0.7257(2)$ & $-0.0598(3)$ & $0.037(1)$ & $0.058(2)$ & $0.062(2)$ & $-0.001(1)$ & $0.005(1)$ & $-0.002(1)$ \\
\hline$C(11)$ & $4 e$ & $0.3864(2)$ & $0.5939(2)$ & $-0.0270(3)$ & $0.044(1)$ & $0.062(2)$ & $0.099(2)$ & $0.017(1)$ & $0.002(1)$ & $0.002(2)$ \\
\hline$C(12)$ & $4 e$ & $0.3336(2)$ & $0.5205(2)$ & $-0.1465(3)$ & $0.062(2)$ & $0.052(2)$ & $0.098(2)$ & $0.010(1)$ & $0.012(2)$ & $-0.009(2)$ \\
\hline$C(13)$ & $4 e$ & $0.2051(2)$ & $0.3420(2)$ & $0.0414(3)$ & $0.056(2)$ & $0.048(2)$ & $0.067(2)$ & $-0.002(1)$ & $0.005(1)$ & $0.006(2)$ \\
\hline$C(14)$ & $4 e$ & $0.2162(2)$ & $0.2105(2)$ & $0.0365(3)$ & $0.095(2)$ & $0.046(2)$ & $0.111(3)$ & $-0.001(1)$ & $0.019(2)$ & $0.017(2)$ \\
\hline$C(15)$ & $4 e$ & $0.5073(2)$ & $0.8230(3)$ & $0.0607(3)$ & $0.043(2)$ & $0.092(2)$ & $0.081(2)$ & $-0.009(2)$ & $0.012(2)$ & $-0.002(2)$ \\
\hline$C(16)$ & $4 e$ & $0.5483(2)$ & $0.8935(3)$ & $0.1844(3)$ & $0.059(2)$ & $0.118(3)$ & $0.087(2)$ & $-0.036(2)$ & $0.003(2)$ & $-0.005(2)$ \\
\hline
\end{tabular}

\section{References}

1. Manta, E.; Scarone, L.; Hernandez, G.; Mariezcurrena, R.; Suescun, L.; Brito, I.; Brouard, I.; Gonzalez, M. C.; Perez, R.; Martin, J. D.: A Facile Synthesis of an Oxatricyclic trans-syn-trans-Substituted Oxepanyl Framework. Tetrahedron Lett. 38 (1997) 5853-5856.

2. Ravelo, J. L.; Regueiro, A.; Rodriguez, E.; Vera, J.; Martin, J. D.: Synthetic studies towards ciguatoxin via acetal/g-oxovynil stannane condensation: a convergent approach. Tetrahedron Lett. 37 (1996) 2869-2872.
3. Alvarez, E.; Candenas, M. L.; Perez, R.; Ravelo, J. L.; Martin, J. D.: Useful designs in the synthesis of trans-fused polyeter toxins. Chem. Rev. 95 (1995) 1953-1980.

4. Sheldrick, G. M.: Phase Annealing in SHELX-90: Direct Methods for Large Structures. Acta Crystallogr. A46 (1990) 467-473.

5. Sheldrick, G. M.: SHELXL-93. Program for the Refinement of Crystal Structures. University of Göttingen, Germany 1993. 